INPLASY

PROTOCOL

To cite: Cheng et al. Should acupuncture therapy be used for acute facial paralysis: a systematic review and metaanalysis of randomised controlled trials. Inplasy protocol 2020100004. doi: 10.37766/inplasy2020.10.0004

Received: 02 October 2020

Published: 02 October 2020

Corresponding author: Lu Cheng

lucheng_2018@163.com

Author Affiliation:

Dongzhimen Hospital

Affiliated to Beijing

University , No. 5,

Haiyangcang, Dongcheng

District, Beijingï $1 / 4$ CEChina

\section{Support: STIPFDZMH}

(DZMKJCX-2020-019).

Review Stage at time of this submission: Preliminary searches.

Conflicts of interest: None.

\section{Should acupuncture therapy be used for acute facial paralysis:
systematic review and meta-analysis of randomised controlled trials

\author{
Cheng, L1; Li, XL2; Mi, SQ3; Zhang, XD4; Guo, W5; Ying, Y6; \\ Zhao, JP7.
}

Review question / Objective: Whether acupuncture should be used in the acute phase of peripheral facial paralysis, and whether an early acupuncture intervention can shorten the recovery time of facial function and reduce the formation of sequelae.

Condition being studied: Facial palsy results in functional and social dysfunction related to the inability to control the muscles of facial expression. Bell's palsy is the most common acute disorder affecting a single nerve and the condition affects $11-40$ people per 100, 000 in the population. Herpes zoster oticus $(\mathrm{HZO})$ is a viral infection of the ear and when associated with acute facial paralysis is known as Ramsay Hunt syndrome. Acupuncture therapy includes acupuncture, moxibustion, bloodletting, fire acupuncture and other treatment methods. Facial paralysis is one of the diseases for which acupuncture treatment has a clear effect that the WHO has clearly announced. However, most patients with bell's palsy show some recovery without intervention within 2-3 weeks after onset of symptoms, and antiviral agents are the standard first-line treatment to reduce or minimise nerve damage. Therefore, whether acupuncture can be performed in the acute phase of facial paralysis and whether the earlier application the better is still controversial.

INPLASY registration number: This protocol was registered with the International Platform of Registered Systematic Review and Meta-Analysis Protocols (INPLASY) on 02 October 2020 and was last updated on 02 October 2020 (registration number INPLASY2020100004).

\section{INTRODUCTION}

Review question / Objective: Whether acupuncture should be used in the acute phase of peripheral facial paralysis, and whether an early acupuncture intervention can shorten the recovery time of facial 
function and reduce the formation of sequelae.

Rationale: Peripheral facial paralysis is one of the indications of acupuncture and moxibustion certified by WHO. It is clinically shown that an early acupuncture in the acute stage of facial paralysis can shorten the recovery time of facial function and reduce the formation of sequelae. At present, there are many clinical studies on the effectiveness of acupuncture treatment of acute facial paralysis, which provides the original data for our systematic review.

Condition being studied: Facial palsy results in functional and social dysfunction related to the inability to control the muscles of facial expression. Bell's palsy is the most common acute disorder affecting a single nerve and the condition affects 11-40 people per 100,000 in the population. Herpes zoster oticus (HZO) is a viral infection of the ear and when associated with acute facial paralysis is known as Ramsay Hunt syndrome. Acupuncture therapy includes acupuncture, moxibustion, bloodletting, fire acupuncture and other treatment methods. Facial paralysis is one of the diseases for which acupuncture treatment has a clear effect that the WHO has clearly announced. However, most patients with bell's palsy show some recovery without intervention within 2-3 weeks after onset of symptoms, and antiviral agents are the standard firstline treatment to reduce or minimise nerve damage. Therefore, whether acupuncture can be performed in the acute phase of facial paralysis and whether the earlier application the better is still controversial.

\section{METHODS}

Search strategy: We searched the following sources from inception to the September 15th 2020:PubMed, the Cochrane Library, the Web of Science, Embase, China National Knowledge Infrastructure (CNKI), Chinese Scientific Journal Database(VIP), Chinese Biomedical database(CBM) and the Wanfang database. Ongoing trials from ClinicalTrials.gov, and the Chinese Clinical Trial Registry(ChiCTR) will also be searched.

Participant or population: There will be no limitation on the populations included in this study, we will include all RCTs of acupuncture for peripheral facial palsy.

Intervention: Acupuncture therapy(including acupuncture, electroacupuncture, moxibustion, blood letting and fire needle around acupoints ) in the acute phase.

Comparator: The commenly used medical therapies (corticosteroids and antiviral medications, etc.), or the same acupuncture treatment as the control group after the acute phase.

Study designs to be included: Randomized controlled trials (RCTs) will be included.

Eligibility criteria: The research object is peripheral facial paralysis or Hunter syndrome in the acute phase, the intervention measures of the treatment group are acupuncture therapies, and the control group is conventional oral drugs (western medicine or traditional Chinese medicine), or placebo with an acupuncture treatment after the acute phase can be included.

Information sources: Eight major English and Chinese electronic databases will be searched: PubMed, Embase, Cochrane Library, Web of Science, CNKI(China National Knowledge Infrastructure), VIP(China Science Technology Journal Database), Wanfang Database、Sino-Med Database (including China Biology Medicine disc(CBM)). Search dates: from their inception to September 15th 2020.

Main outcome(s): The recovery rate of facial function, how long it takes to restore facial function and the odds of sequelae occurring. (use House-Brackman scales, Facial Nerve Grading 2.0 and other related scales). 
Additional outcome(s): Psychological wellbeing and quality of life.(use Facial Disability Index scale (FDI), World Health Organization Quality of Life Scale-Brief Form Questionnaire (WHOQOL-BREF)).

Quality assessment / Risk of bias analysis: Two reviewers will evaluate the quality of the selected studies independently according to the Cochrane Collaboration's tool for randomized control trials. The following characteristics will be assessed: sequence generation (selection bias), allocation concealment (selection bias), blinding of participants and personnel (performance bias), blinding of outcome assessment (detection bias), incomplete outcome data (attrition bias), selective reporting (reporting bias), and other sources of bias. These questions will be assessed by Review Manager 5.3.

Strategy of data synthesis: If studies are judged to be homogeneous, we will perform a meta-analyses. We will pool the data using risk ratio or odds ratio with $95 \%$ confidence interval $(\mathrm{Cl})$ for dichotomous outcomes, as for continuous outcomes, we will use standard mean difference or mean difference with $95 \% \mathrm{Cl}$. The clinical heterogeneity will be assessed according to the characteristics of the included studies and participants, details of the intervention or control, types of outcome measurements will also be analysed. The $\mathbf{I}^{2}$ statistic will be used to assess the heterogeneity. According to the cochrane handbook, when $\mathrm{I}^{2}$ is between $0 \%-40 \%$, $30 \%-60 \%, 50 \%-90 \%, 75 \%-100 \%$, the heterogeneity will be regarded as not important, moderate, substantial and considerable. We will use random-efects model to conduct the meta-analysis unless the $I^{2}$ statistic is $75 \%$. When there is a substantial heterogeneity, we will try to investigate possible causes from clinical perspectives. Subgroup and sensitivity analysis will be conducted. Forest plots will show the results of the meta-analysis, and if there are more than $\mathbf{1 0}$ included studies, a funnel plots willed be used to identify publication bias.
Subgroup analysis: For facial paralysis, there are different severity and TCM syndrom; for intervention, there are different type of acupuncture therapy; and there are different type of control group. These all can lead to heterogeneity. In order to explore the treatment effects respectively, we plan to conduct subgroup analysis for different severity, syndroms, acupuncture techniques (such as manuacupuncture, electroacupuncture, a combination of different method, different stimulate of acupoint, different acupoint selection), control group and other possible factors.

Sensibility analysis: For the main outcome with important positive significance, when the literature conditions are met, the random method is compared according to the methodological quality of the literature. Clear/unclear, double-blind use or not; when the combined results are in a critical state and the heterogeneity is small, compare results of random effects model and fixed effects model. If necessary,leave each individual study out to assess the weight of the research.

Language: No restriction.

Country(ies) involved: China.

Keywords: Systematic review, Metaanalysis, complementary therapy, acupuncture, acupuncture therapy, peripheral facial paralysis, facial palsy, physiotherapy.

Contributions of each author:

Author 1 - Lu Cheng.

Author 2 - Xiaolin Li.

Author 3 - Shuqi Mi.

Author 4 - Xudong Zhang.

Author 5 - Wei Guo.

Author 6 - Yi Ying.

Author 7 - Jiping Zhao. 